Original Paper

http://indexmedicus.afro.who.int

\title{
Tomato pomace powder ameliorated cisplatin-induced microanatomical alterations in brain of Wistar rats
}

\author{
Olatunde OWOEYE ${ }^{1 *}$ and Silas Kalu ONWUKA ${ }^{2}$ \\ ${ }^{1}$ Department of Anatomy, College of Medicine, University of Ibadan, Ibadan, Nigeria. \\ ${ }^{2}$ Department of Veterinary Anatomy, Faculty of Veterinary Medicine, University of Ibadan, Nigeria. \\ *Corresponding author; E-mail: o.owoeye@mail.ui.edu.ng; oowoeye2001@yahoo.com;
} Tel: +234-8033239973; Fax: 234-2-8103043

\begin{abstract}
Cisplatin chemotherapy is associated with neurotoxicity. Oxidative stress has been associated with its pathogenesis. Tomato (Lycopersicon esculentum) pomace powder (TPP) may be a preventive agent by virtue of its known antioxidant property. The possible protective role of TPP against cisplatin-induced alteration of the microanatomy of rat brain was investigated. Thirty rats were divided equally into five groups: control, propylene glycol, TPP, cisplatin (10 mg/kg i.p.); TPP $50 \mathrm{mg} / \mathrm{kg}$ plus cisplatin $(10 \mathrm{mg} / \mathrm{kg}$ i.p.). All administrations were oral by gastric gavage given for 24 days except cisplatin which was given intraperitoneally on days 10 and 17 . Rats were euthanized on the $25^{\text {th }}$ day of treatment. Blood parameters checked, neurobehavioural tests done and brain tissue were examined with regards to micro-anatomical parameters. Cisplatin caused a significant $(\mathrm{p}<0.05)$ reduction in lymphocytes. Neurobehavioural results showed that cisplatin caused a reduction in line crossing, rearing and forelimb grip. Cisplatin caused histological alterations in the cerebellum, dentate gyrus and Cornu Ammonis3 (CA3). Co-treatment of cisplatin with TTP ameliorated these haematological, neurobehavioural and histological alterations. In conclusion, Lycopersicon esculentum (as tomato pomace powder) demonstrated neuroprotection against cisplatin-induced alteration of microanatomy of rat cerebellum, dentate gyrus and Cornu Ammonis3 of rat brain.
\end{abstract}

(C) 2015 International Formulae Group. All rights reserved.

Keywords: Lycopersicon esculentum, cisplatin, neuroprotection, Cornu Ammonis3, dentate gyrus, cerebellum.

\section{INTRODUCTION}

Cisplatin, cis-diamminedichloroplatinum with the molecular formula cis[ $\mathrm{Pt}(\mathrm{NH} 3) 2 \mathrm{Cl} 2]$, is a platinum-based drug used as an antineoplastic agent which has unwanted and sometimes severe side effects limiting its usage. Therefore, methods that may reduce these side effects are worth investigating. It is a widely used and effective cytotoxic agent in the treatment of a variety of malignant tumours, including lung, colorectal, ovarian, breast, head and neck, bladder and testicular cancers in both children and adults (Gulec et al., 2013). Despite being a commonly used chemotherapy drug, the benefit of cisplatin is compromised by severe side effects including neurotoxicity, myelotoxicity, nephrotoxicity, ototoxicity and haematological toxicity (McWhinney et al., 2009).

Cisplatin-induced neurotoxicity is reported to occur in a cumulative and dosedependent (Amptoulach and Tsavaris, 2011), 
but there have been reports that single doses of cisplatin administration elicited toxicities in different organs of laboratory animals (Fahmy et al., 2013; Gulec et al., 2013). Cisplatinmediated neurotoxicity is a significant limitation to effective anti-cancer treatment because it may lead to dose reduction or even treatment withdrawal (Nowis et al., 2007). Despite the report that platinum-based agents have poor penetration through the blood-brain barrier (McKeage et al., 2001), the central nervous system has not been spared from its toxicity which has been partially attributed to different causes, one of which is oxidative damage (Gulec et al., 2013; Turan et al., 2013). Evidence has shown that cisplatin may act on the mitochondria thus inducing mitochondrial dysfunction probably through binding of cisplatin to mitochondrial DNA (Carozzi et al., 2009). Mitochondrial dysfunction would increase oxidative stress which plays an important role in the pathogenesis of cisplatin neurotoxicity. Indeed, antioxidant treatments have been used successfully in order to reduce cisplatinmediated neurotoxicity as demonstrated by Turan et al. (2013) and Gulec et al. (2013) who showed that cisplatin significantly increased the levels of oxidant parameters such as lipid peroxidation (thio-barbituric acid reactive substance (TBARS), myeloperoxidase (MPO) and 8hydroxyguanine (8-OH-GUA) in brain tissue and suppressed the effects of antioxidants such as total glutathione (GSH) and superoxide dismutase (SOD). The use of naturally occurring antioxidant that are plantbased, are being encouraged hence the interest in the reports showing the reduction of toxicity of cisplatin by natural substances (AlMoundhri et al., 2013; Pandir and Kara, 2013). Tomato or Lycopersicon esculentum Mill has been reported to contain lycopene, an effective antioxidant (Wattanathorn et al., 2012). Tomato pomace powder (TPP) made from tomato has been beneficial in protecting against experimental stroke (Wattanathorn et al., 2012). Tomato belongs to the family of Solanaceae, and phytochemical investigations have shown that one of its constituent, lycopene is a potent carotenoid antioxidant which provided protection from focal ischaemic cerebral injuries (Hsiao et al., 2007).

It is well known that the cerebral hemisphere, hippocampus and cerebellum are responsible for regulating cognition and primary sensory functions; memory storage; motor coordination, balance and postural stability functions respectively. Any of these central nervous system components may be affected by drug induced toxicity resulting in altered anatomy and physiology of affected parts of the central nervous system. Literature is scanty concerning the role of TPP on cisplatin-induced alteration of the microanatomy of rats brain hence this study of a possible protective role utilizing its known antioxidant property.

This study aimed at assessing the protective effect of tomato pomace powder against cisplatin-induced neuropathy in rats.

\section{MATERIALS AND METHODS Experimental animals}

This study was carried out using thirty male Wistar rats with initial weight $77-84 \mathrm{~kg}$, which were housed in the Animal House, of the Department of Veterinary Physiology, Biochemistry and Pharmacology, University of Ibadan, Nigeria. They were acclimatized to laboratory room conditions (12 hours dark light period) for a week before the onset of experiment. The rats were fed with rat chow from Ladokun Feeds, Ibadan, Nigeria and water ad libitum. The experimental protocols were carried out according to the approval and guidelines set by the University of Ibadan Ethical Committee which also conformed to the acceptable guidelines on the ethical use of animals in research (Public Health Service, 1996). 


\section{Tomato Pomace Powder (TPP) Preparation and Administration}

Fresh tomato fruits (Lycospersicum esculentum) were purchased from Bodija market, Ibadan, Nigeria. TPP was prepared according to the published method of Wattanathorn et al. (2012). Briefly, the fresh tomatoes were washed and cut into small pieces after which the tomato juice was extracted to retain the skin, pulp and seeds which were then dried in an oven at $50{ }^{\circ} \mathrm{C}$ for 2 hours. The dried parts were weighed and grinded in a blender and the resulting powder termed Tomato Pomace Powder (TPP) was kept in a clean airtight plastic container at room temperature till ready for use. The administration of TPP was performed by using propylene glycol as vehicle.

\section{Chemicals}

Cisplatin was procured from Korea United Pharm. Inc. (Naojang, Chungnam, Korea) and propylene glycol from Guangdong Guanghua Science Tech. Co Ltd. (China). Ketamine was manufactured by Rotex Medica, Trittau, Germany. All other reagents were of analytical grade and were obtained from the British Drug Houses (Poole, Dorset, UK).

\section{Experimental design}

After the period of acclimatization, the thirty male rats were randomized into 5 groups of 6 rats each with groupings as below in Table 1:

Cisplatin was given i.p. while all other treatments were given orally with a gavage. The $\mathrm{LD}_{50}$ of cisplatin in rats is $12 \mathrm{mg} / \mathrm{kg}$ (IARC, 1981). The dose and route of administration of the single doses of cisplatin was based on the methods of Gulec et al. (2013) and Fahmy et al. (2013), while that of TPP was based on the method of Wattanathorn et al. (2012).

\section{Neurobehavioural tests}

On day 25 of the experiment, we investigated the effects of cisplatin toxicity on the rat nervous system, by performing some behavioural tests for all the animals. They were initially weighed, and then subjected to neurological tests namely: (1) wire grip test, (2) negative geotaxis, and (3) open field test.

\section{Wire grip test}

This test was performed according to a modification of the method of Van Wijk et al. (2008). Each rat was suspended with both forepaws on a horizontal steel wire ( 1 meter long, diameter $7 \mathrm{~mm}$ ). The animal was held in a vertical position when its front paws were placed in contact with the wire. When the rat grasped the wire, it was released, and the latency to fall was recorded with a stopwatch. Rats were randomly tested and each animal was given three trials with a 30 min inter trial rest interval. This test assessed muscle strength and balance.

\section{Negative geotaxis}

Negative geotaxis was tested by placing rats head-down on an inclined plane and then watched the rat orient in a head-up direction (Kreider and Blumberg, 1999). The time it takes for the rat to orient in a head-up direction was recorded with a stopwatch.

\section{Open field test}

The apparatus used was a slight modification of the method of Mohammad et al., (2010). It consisted of a square arena $(56 \times 56 \times 20 \mathrm{~cm})$ made of white wood and its floor divided by lines into 16 squares that allowed the definition of central and peripheral parts. At the beginning of the session, each rat was individually placed in the centre of the arena and its activity was recorded for $5 \mathrm{~min}$. The number of squares crossed with all paws (crossing) and standing on legs (rearing) were evaluated during 5 minute sessions. The crossing numbers were indicators of locomotor while the rearing numbers indicated vertical and exploratory activities. At the end of each session, rats were removed from the openfield and the experimental chamber was thoroughly cleaned with a damp cloth and dried.

\section{Sample collection and histological preparation}

After the neurological tests, blood was collected from each rat via the retro-orbital plexus into lithium heparinized bottles for 
haematological indices. Thereafter, the rats were euthanized by cervical dislocation after sedation with intraperitoneal injection of 10 $\mathrm{mg} / \mathrm{k}$ of Ketamine. Each rat was decapitated at the cervico-medullary junction for uniformity and the skulls opened after which the brains were quickly extracted. After washing gently with saline and weighing the whole brain, the brain was fixed in $10 \%$ neutral buffered formalin for three days and was consequently used for histology. The cerebellum, cerebral cortex and hippocampus of each animal were dissected and then preserved for paraffin embedment technique.

\section{Histology}

The cerebellum from each group was obtained and homologous sampling was assured by obtaining transverse sections of the right cerebellum from each specimen from the lateral zone portions of the cerebella hemisphere (vermal, paravermal and flocullus portions were not utilized) for uniformity. Coronal sections of the right half of each brain were made to obtain samples of the frontal cortex and hippocampal tissue. The tissues were sectioned at 5-6 $\mu \mathrm{m}$ thickness and then stained with Haematoxylin and Eosin according to the method of Bancroft and Gamble, (2008). Following mounting and staining, slides were examined on an Olympus $\mathrm{CH}$ (Japan) light microscope and photomicrographs acquired with a Sony DSCW 3 digital camera (Japan).

\section{Determination of haematological values}

K2 EDTA-added whole blood samples were used for hematological analyses immediately after collection with the aid of Sysmex Automated Hematology (KX-21, Kobe, Japan) Analyzer. The haematocrit or packed cell volume (PCV), haemoglobin $(\mathrm{Hb})$, red blood cell count (RBC), mean cell volume (MCV), mean cell haemoglobin $(\mathrm{MCH})$, and mean cell haemoglobin concentration (MCHC) and white blood cell count (WBC), were obtained.

\section{Statistical analysis}

All data were expressed as means \pm standard deviation. Data were analysed using one-way analysis of variance (ANOVA) using
GraphPad Prism TM 4.0 version software, San Diego, CA, USA. Post hoc comparisons were performed after ANOVA using Dunnett's test. Statistical significance was set at $\mathrm{p}<0.05$.

\section{RESULTS}

\section{Haematological parameters}

Table 2 summarizes the effect of $\mathrm{TPP}+\mathrm{Cis}$ on erythrocyte indices of male Wistar rats. It was observed that there was no effect of either TPP or cisplatin on the packed cell volume (PCV), haemoglobin level ( $\mathrm{HgB})$, total red blood cell count (RBC), mean corpuscular volume (MCV), and mean corpuscular haemoglobin concentration (MCHC) of the rats. The effect of cisplatin and tomato pomace powder on the Leukocyte indices of male Wistar rats is presented in Table 3. Although there was no significant change in the total white blood cell (WBC), cisplatin caused a significant reduction $(p<0.05)$ of the neutrophil count of the rats, but an elevation of the eosinophils.

\section{Neurobehavioural parameters}

Shown in Table 4 is a summary of the effect of $\mathrm{TPP}+\mathrm{Cis}$ on the behavioural and locomotor activities of male Wistar rats. The number of lines crossed and rearing were significantly reduced in all treated groups $(p<0.05)$. However, there were significant increases in the TPP+Cis group compared with the cisplatin only group. There was a significant increase in the latency of forelimb wire grip test $(\mathrm{p}<0.05)$.

\section{Histological parameters Cerebellum}

The cerebellum of the control rats showed the normal histological features of 3 layers: molecular, Purkinje and granular as shown in Figure 1a. The effect of cisplatin is shown in Figure 1b where the Purkinje cells of the cerebellum exhibited eosinophilia unlike in other groups showing the normal basophilia. Figures 1c, 1d and 1e show cerebellar architecture similar to control.

\section{Cerebral cortex}

The cerebral cortex of the control rats showed the normal histological features with 
the cortical neurons showing round or ovoid nuclei exhibiting dispersed chromatin as shown in the different treatment groups. This is evident in Figures 2a, 2b, 2c, 2d, and 2e. The features are similar to those of control brain.

\section{Dentate gyrus}

The dentate gyrus of the hippocampal formation of the control rats showed the normal histological features of the molecular layer, granule cell layer and the polymorphic layer. Of interest was the granule cell layer consisting of several layers of granule cells with large nuclei exhibiting open chromatin as shown in Figure 3a, 3b, and 3c. The effect of cisplatin is shown in Figure $3 d$ where the granule cell neurons show evidence of toxicity as exhibited by many pyknotic neurons (arrow). Affected are the neurons of the subgranular zone of the dentate gyrus. Figure $3 \mathrm{e}$ shows some ameliorative effect of cotreatment of cisplatin with the TPP. The pyknotic neurons are very much reduced when compared with the cisplatin treated brains.

\section{Cornu Ammonis3 (CA3)}

The CA3 subfield of the hippocampal formation of the control rats showed portions of the normal histological features of the stratum oriens, pyramidal cell layer, stratum radiatum, all of which show normal cytoarchitecture as shown in Figure 4a. Of special note are the pyramidal neurons in layers with nuclei exhibiting open chromatin as shown in Figure 4a, 4b, and 4c. In Figure $4 \mathrm{~d}$, cisplatin-toxicity on the pyramidal neurons was exhibited by neuronal degeneration (arrows) shown by pyknosis and angulated neurons. Figure 4e shows the effect of co-treatment of cisplatin with the TPP showing few scattered pyknotic neurons of the pyramidal cell layer when compared with the cisplatin treated brains.

Table 1: Experimental design.

\begin{tabular}{ll}
\hline Grouping & Treatment \\
\hline Group I & Control, received rat chow and water daily. \\
\hline Group II & Propylene glycol (PG), received $0.2 \mathrm{~mL}$ daily of the vehicle for 24 days. \\
\hline Group III & $\begin{array}{l}\text { Tomato Pomace Powder (TPP), received a daily treatment of TPP dose of } 50 \mathrm{mg} / \mathrm{kg} \\
\text { bwt for } 24 \text { days. }\end{array}$ \\
\hline Group IV & Cisplatin (Cis), received $10 \mathrm{mg} / \mathrm{kg} \mathrm{bwt} \mathrm{(i.p.)} \mathrm{on} \mathrm{days} 10$ and 17 of the experiment \\
\hline Group V & $\begin{array}{l}\text { TPP+Cis, received Cisplatin } 10 \mathrm{mg} / \mathrm{kg} \text { bwti.p. on days } 10 \text { and } 17 \text { of the experiment }+ \\
\text { TPP 50 } \mathrm{mg} / \mathrm{kg} \text { for } 24 \text { days. }\end{array}$ \\
\hline
\end{tabular}

Table 2: Effect of cisplatin and tomato pomace powder on erythrocyte indices of male Wistar rats.

\begin{tabular}{lccccc}
\hline Groups & $\begin{array}{c}\text { PCV } \\
(\boldsymbol{\%})\end{array}$ & $\begin{array}{c}\mathbf{H g B} \\
(\mathbf{g} / \mathbf{d L})\end{array}$ & $\begin{array}{c}\mathbf{R B C} \\
(\mathbf{x 1 0} / \boldsymbol{\mu L})\end{array}$ & $\begin{array}{c}\text { MCV } \\
(\mathbf{f L})\end{array}$ & MCHC (g/dL) \\
\hline Control & $46.00 \pm 1.41$ & $15.10 \pm 0.42$ & $7.48 \pm 0.07$ & $61.00 \pm 1.41$ & $33.33 \pm 0.58$ \\
PG & $45.00 \pm 3.0$ & $15.53 \pm 0.85$ & $7.44 \pm 0.51$ & $60 \pm 2.08$ & $34.00 \pm 0.58$ \\
TPP & $43.50 \pm 2.19$ & $14.50 \pm 1.02$ & $7.50 \pm 1.63$ & $59.50 \pm 2.12$ & $33.00 \pm 0.00$ \\
Cis & $42.67 \pm 1.65$ & $13.10 \pm 1.29$ & $6.37 \pm 2.40$ & $60.00 \pm 2.00$ & $32.50 \pm 0.71$ \\
TPP+Cis & $44.00 \pm 2.54$ & $14.60 \pm 1.39$ & $7.07 \pm 1.38$ & $61.67 \pm 1.15$ & $32.50 \pm 0.71$ \\
\hline
\end{tabular}

Data are expressed as mean \pm standard deviation of six animals. Cis, cisplatin; PG, propylene glycol; TPP, tomato pomace powder. 
Table 3: Effect of cisplatin and tomato pomace powder on the Leukocyte indices of male Wistar rats.

\begin{tabular}{lccccc}
\hline Groups & $\begin{array}{c}\text { WBC } \\
\left(\mathbf{x 1 0}^{3} / \boldsymbol{\mu L}\right)\end{array}$ & $\begin{array}{c}\text { Lymphocytes } \\
(\mathbf{x 1 0} / \boldsymbol{\mu} \mathbf{L})\end{array}$ & $\begin{array}{c}\text { Neutrophils } \\
\left(\mathbf{x} 1 \mathbf{3}^{\mathbf{3}} / \boldsymbol{\mu L}\right)\end{array}$ & $\begin{array}{c}\text { Monocytes } \\
(\mathbf{x 1 0} \boldsymbol{3} / \boldsymbol{\mu L})\end{array}$ & $\begin{array}{c}\text { Eosinophils } \\
(\mathbf{x 1 0} / \boldsymbol{\mu} \mathbf{L})\end{array}$ \\
\hline Control & $5.58 \pm 1.40$ & $2.13 \pm 09.44$ & $3.81 \pm 1.2$ & $1.55 \pm 0.40$ & $0.00 \pm 0.00$ \\
PG & $6.80 \pm 1.71$ & $2.35 \pm 1.16$ & $2.24 \pm 0.7$ & $1.11 \pm 0.3$ & $1.06 \pm 0.12$ \\
TPP & $3.23 \pm 0.38$ & $2.50 \pm 0.48$ & $1.07 \pm 1.01$ & $1.31 \pm 0.2$ & $2.13 \pm 0.18$ \\
Cis & $4.07 \pm 1.31$ & $2.23 \pm 0.19$ & $1.73 \pm 0.6^{*}$ & $1.11 \pm 0.24$ & $2.4 \pm 1.60^{*}$ \\
TPP+Cis & $4.83 \pm 08.29$ & $2.63 \pm 07.00$ & $2.74 \pm 0.68$ & $1.32 \pm 02$ & $1.70 \pm 1.73$ \\
\hline
\end{tabular}

Data are expressed as mean \pm standard deviation of six animals. Cis, cisplatin; PG, propylene glycol; TPP, tomato pomace powder. ${ }^{*} \mathrm{P}<0.05$ versus Control group; $* *=\mathrm{P}<0.05$ versus cisplatin group.

Table 4: Effect of cisplatin and tomato pomace powder on behavioural and locomotor activities of male Wistar rats.

\begin{tabular}{lcccc}
\hline Groups & Line Crossing & Rearing & Forelimb & Geotaxis \\
\hline Control & $46.20 \pm 5.89$ & $17.00 \pm 4.51$ & $5.67 \pm 2.31$ & $1.60 \pm 0.55$ \\
PG & $23.67 \pm 11.16^{*}$ & $11.25 \pm 2.87$ & $4.80 \pm 1.79$ & $2.50 \pm 1.05$ \\
TPP & $25.00 \pm 4.97^{*}$ & $8.33 \pm 1.53$ & $4.83 \pm 0.75$ & $2.67 \pm 1.63$ \\
Cis & $20 \pm 11.53^{*}$ & $5.50 \pm 3.70^{*}$ & $4.00 \pm 1.41$ & $2.25 \pm 0.50$ \\
TPP+Cis & $25.33 \pm 15.03^{* *}$ & $14 \pm 11.31^{* *}$ & $8.33 \pm 0.58^{* *}$ & $3.50 \pm 2.12$ \\
\hline
\end{tabular}

Data are expressed as mean \pm standard deviation of six animals. Cis, cisplatin; PG, propylene glycol; TPP, tomato pomace powder. $* \mathrm{P}<0.05$ versus Control group; $* *=\mathrm{P}<0.05$ versus cisplatin group.
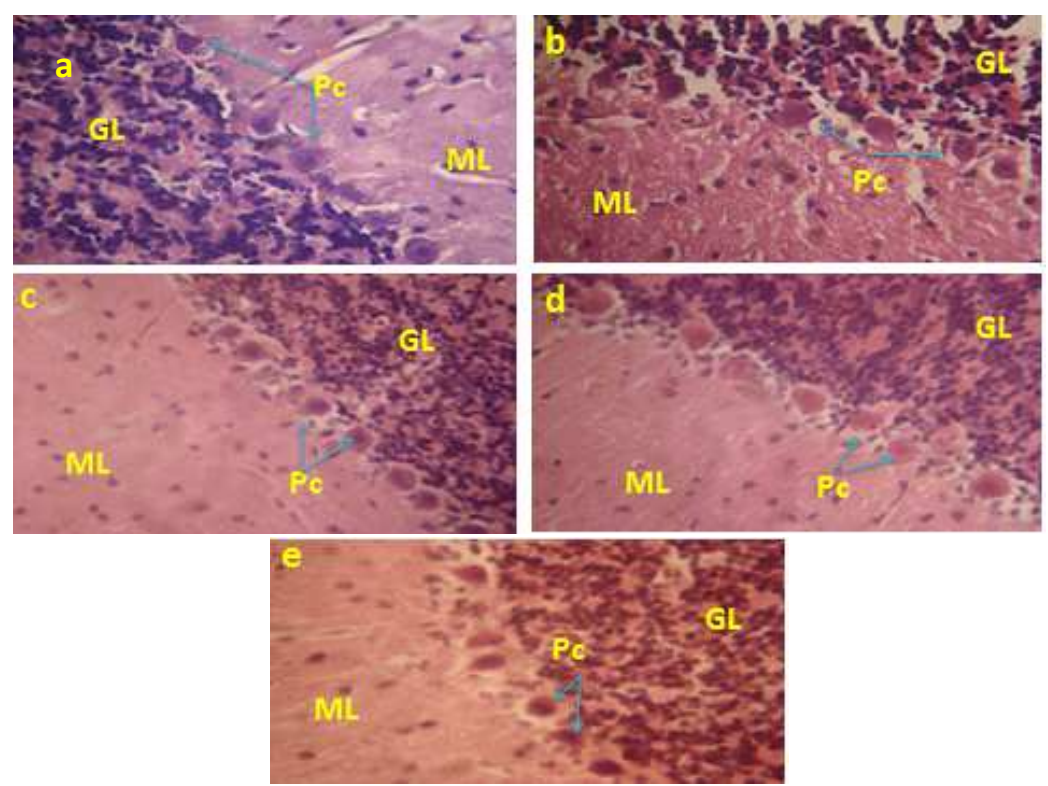

Figure 1: Representative stained sections of cerebellum of rats: (a) Control rats, (b) PG-treated, (c) TTP-treated, (d) Cis-treated cerebellum with the Purkinje neurons exhibiting eosinophilia, and (e) TPP+Cis-treated. Purkinje cells show normal basophilic staining nuclei in all groups except in Cistreated group. PG, propylene glycol; TTP, tomato pomace powder; Cis, cisplatin; ML : molecular layer; GL : granular layer; Pc : Purkinje cells. H\&E. x 960. 

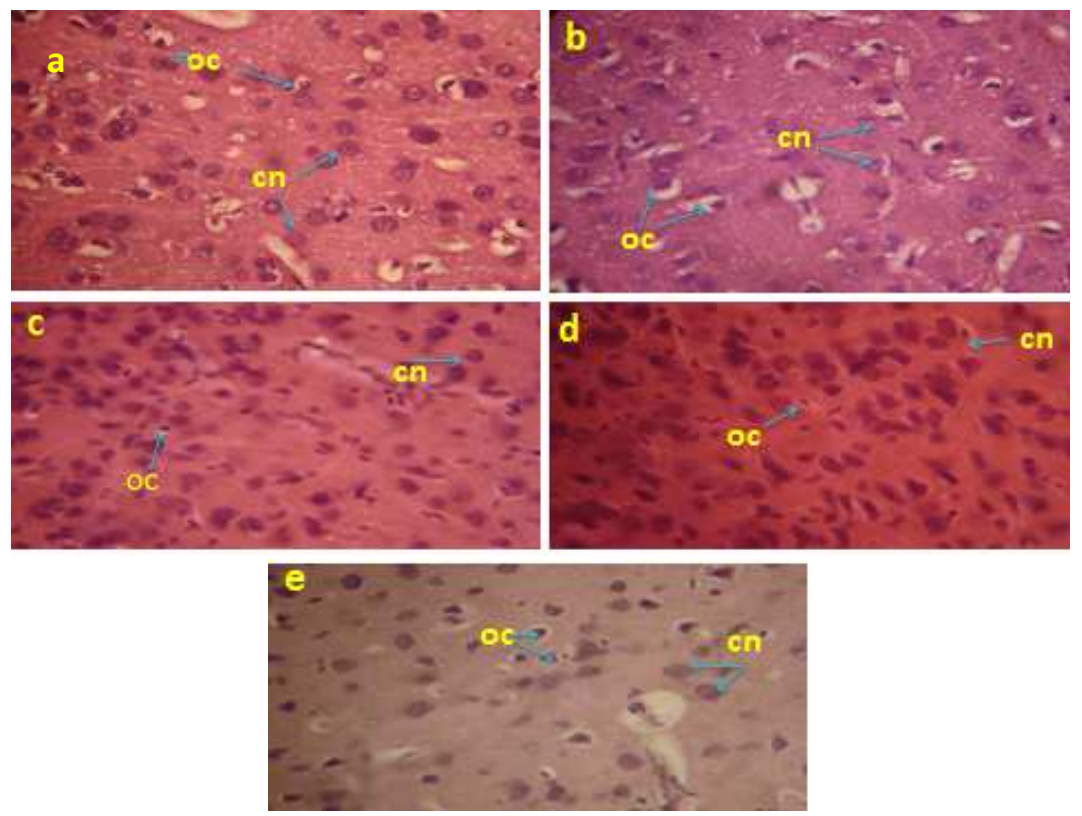

Figure 2: Representative stained sections of cerebral cortex of rats: (a) Control rats, (b) PG-treated, (c) TTP-treated, (d) Cis-treated and (e) TPP+Cis-treated. PG, propylene glycol; TTP, tomato pomace powder; Cis, cisplatin; cn, cortical neuron; oc, oligodendrocyte. H\&E. x 960.
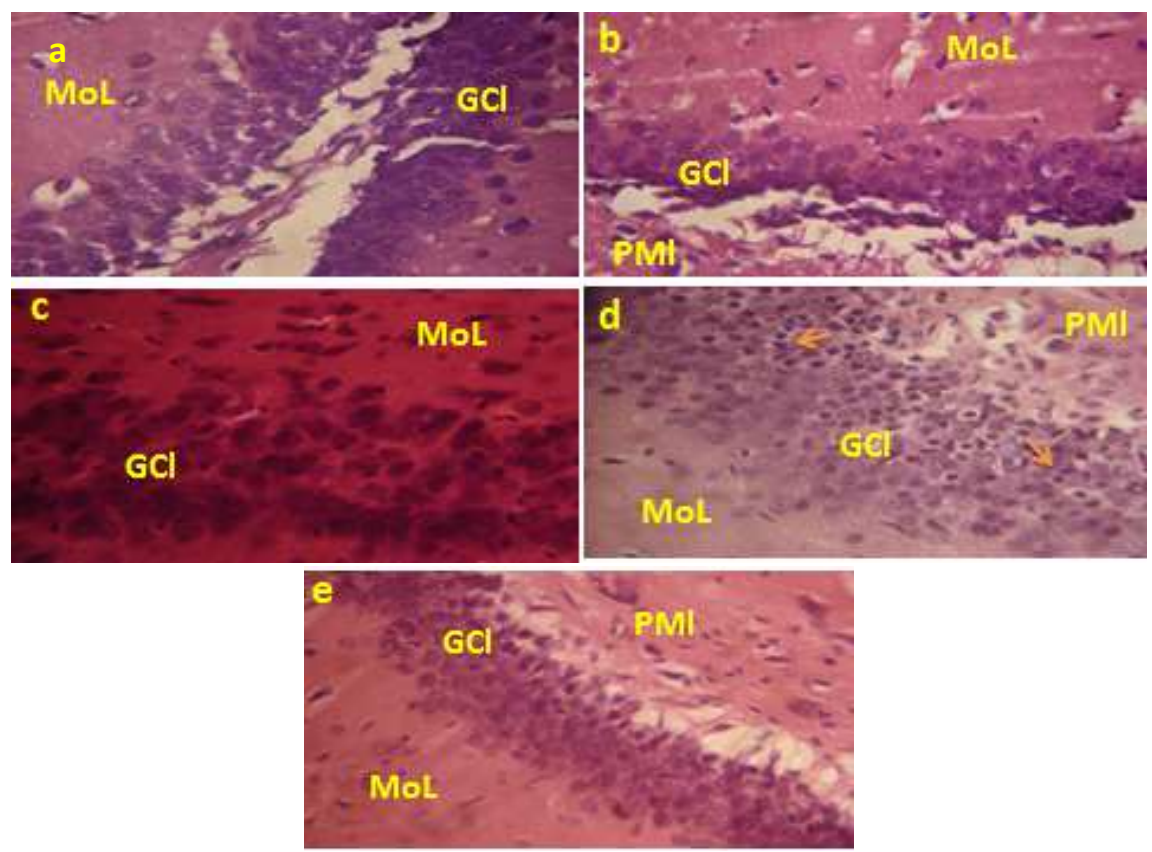

Figure 3: Representative stained sections of dentate gyrus of rats. (a) Control rats, (b) PG-treated, (c) TTP-treated, (d) Cis-treated group shows scattered pyknotic neurons in $\mathrm{GCl}$ (arrows), and (e) TPP+Cis-treated rats show fewer scattered pyknotic neurons. PG, propylene glycol; TTP, tomato pomace powder; Cis, cisplatin; MoL, molecular layer; GCl, granular cell layer; PMl, polymorphic layer. H\&E. x 960. 

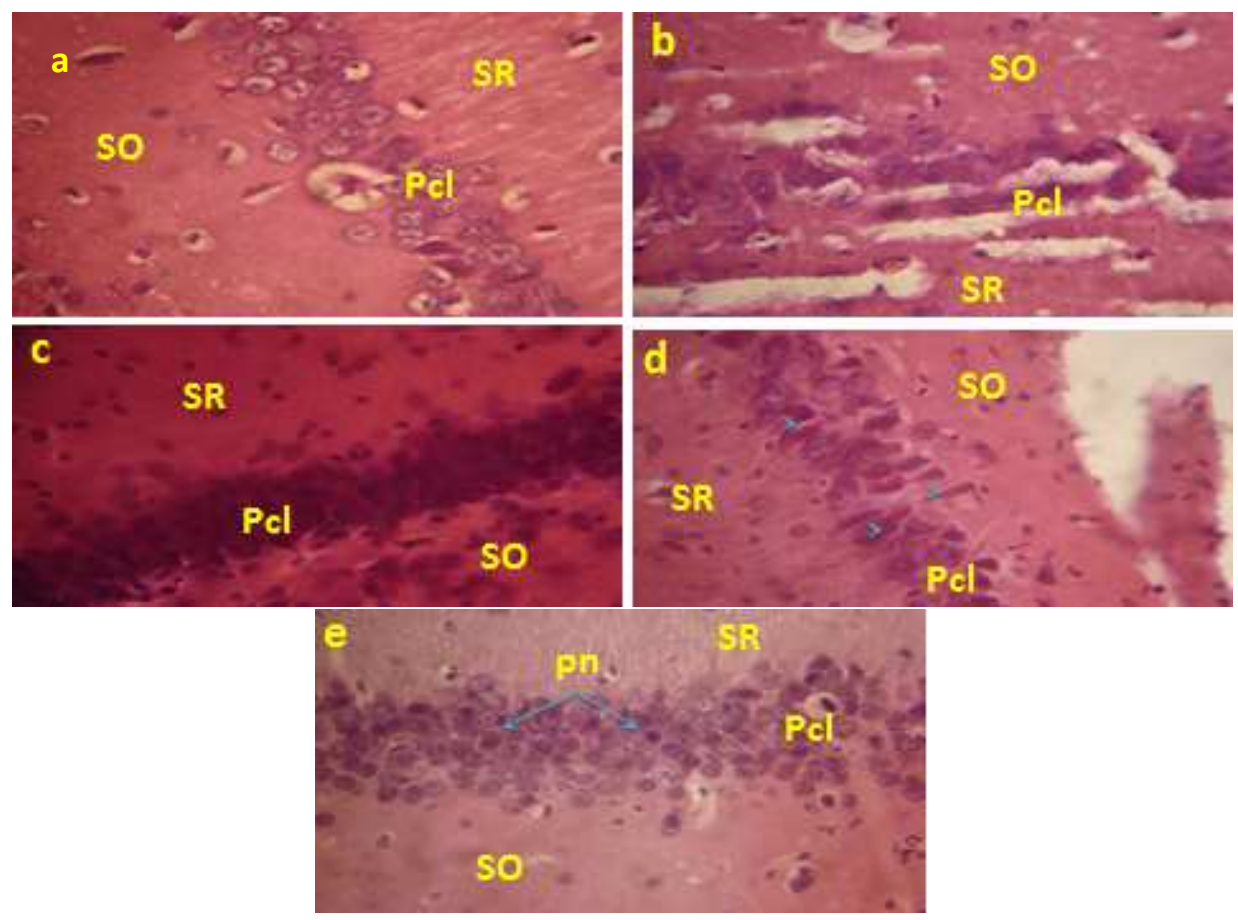

Figure 4: Representative stained sections of Cornu Ammonis 3 of rats. (a) Control rats, (b) PGtreated, (c) TTP-treated, (d) Cis-treated rats show degenerating and angulating pyramidal neurons and (e) TPP+Cis-treated rats showing fewer scattered pyknotic pyramidal neurons. PG, propylene glycol; TTP, tomato pomace powder; Cis, cisplatin; SO, stratum oriens layer; Pcl, pyramidal cell layer; SR, stratum radiatum; pn, pyknotic neuron; blue arrowheads, degenerating neuron. H\&E. x 960.

\section{DISCUSSION}

The potential protective effects of Lycopersicon esculentum as pomace powder (TPP) on cisplatin-toxicity on the central nervous system of male rats was investigated. It was found that tomato ameliorated the haematological, neurobehavioural and the histomorphological alterations induced by cisplatin.

Several workers have consistently reported that the mechanism of cisplatin toxicity was through oxidative stress (Turan et al., 2013; Gulec et al., 2013; Fahmy et al., 2013; Prabhu et al., 2013). The biochemical results of these workers have shown the generation of free radicals in each case as they demonstrated that cisplatin treatment was characterized by a significant increase in tissue malon-dialdehyde level compared to normal control (Ilbey et al., 2009). This study investigated the associated response of neural and other parameters in response to cisplatin toxicity. Bottone et al. (2012) had reported that cisplatin induces cell death in vivo and in vitro in cerebellum and hippocampus of rats. Kim et al. (2010) presumed that irreversible cell damage caused by cisplatin might result mainly from oxidative stress.

Our findings that cisplatin-induced neurotoxicity was associated with histological alteration agreed with the reports of the reports of Al Moundhri et al. (2013) and Arrieta et al. (2011) who similarly reported this observation. This supported our findings of cisplatin's alteration of the histology of the densely packed layers of granule neurons of the dentate gyrus of the hippocampal formation and the pyramidal neurons of the CA3 zone of the hippocampus proper when compared with the neurons of the control rats. 
Of importance are the cells of hilar and subgranular zone (SGZ) of the granule cell layer of the dentate gyrus exhibiting cellular death. Similarly, cisplatin also caused complete dissolution of the basophilic materials of the nuclei of Purkinje cells of the cerebellum of the rats showing eosinophilia. This agrees with the report of Pisu et al. $(2004,2005)$ that cisplatin has negative effects on cerebellar cortex neurons, especially Purkinje cells. Although platinum drugs are reported to have poor penetration of the blood-brain barrier (Gregg et al., 1992), sufficient levels of cisplatin have been shown to cause toxicity in the brain (Gulec et al., 2013), which this study demonstrated.

By implication, this cisplatin-induced Purkinje cell alteration may lead to cerebellar injury which may cause gait, movement and posture impairments (Pisu et al., 2004). Similarly, the histological alterations caused by neuronal death as observed in the dentate gyrus neurons and those of the pyramidal cells of Cornu Ammonis3 might disrupt the smooth flow of neural information from the entorhinal cortex to the granular layer of dentate gyrus and further propagation of mossy fibres to the CA3 and CA1 subzones of the hippocampus as reported by Vivar and Praag (2013). With evidence of death of pyramidal neurons of $\mathrm{CA} 3$, the subsequent projection of impulses from CA3 via the Schaffer's fibres to CA1 may be affected further altering the flow of neural information. Also, adult neurogenesis may be affected due to the neuronal death shown in the SGZ of the granule cell layer of the dentate gyrus. In essence, this suggests that memory and other postulated hippocampal functions might potentially be affected in such rats (Vivar and van Praag, 2013).

However, antioxidants are known to ameliorate the effect of oxidative stress. Phytochemical investigation of tomato has shown that one of its constituent, lycopene is a potent carotenoid antioxidant which provided protection from focal ischaemic cerebral injuries (Hsiao et al., 2007). Lycopene has been reported to scavenge singlet molecular oxygen and peroxyl radicals thus contributing to the defence against lipid peroxidation (Stahl and Sies, 2003). Tomato pomace powder (TPP) made from tomato has been beneficial in protecting against experimental stroke (Wattanathorn et al., 2012). Similarly, the enhancement by TPP of the levels of the neutrophils and eosinophils suggests an improved status of the haematological function.

Co-treatment of cisplatin with TPP demonstrated neuroprotection of the Purkinje neurons of the cerebellum, and partial histomorphological protection for the dentate granule cells of dentate gyrus. Similarly, TPP protected the pyramidal neurons of the Cornu Ammonis 3 of the hippocampus as demonstrated in the improvement in the histological features observed in the treated brains. The neurobehavioural test results also suggested that TPP contributed to the improvement of the crossing numbers, indicators of locomotor activity, rearing numbers indicating exploratory activity, and the forearm grip implying improved muscular strength. These suggest that TPP could offer some measure of neuroprotection against cisplatin-induced neurotoxicity most likely through its reported antioxidant property.

\section{Conclusion}

The findings reported in the study indicate that the oral administration of Lycopersicon esculentum as tomato pomace powder ameliorated intraperitoneally administered cisplatin-toxicity on the cerebellum, dentate gyrus and Cornus Ammonis3 of rats' brain thus exhibiting significant neuroprotective effect.

\section{ACKNOWLEDGMENTS}

The valuable review and comments of Prof. V. O. Taiwo, Head of Department of Veterinary Pathology, University of Ibadan on the histological slides are greatly acknowledged and appreciated. 


\section{REFERENCES}

Al Moundhri MS, AL-Salam S, Al Mahrouqee A, Beegam MS, Ali BH. 2013. The effect of curcumin on oxaliplatin and cisplatin neurotoxicity in rats: Some behavioural, biochemical, and histopathological studies. J. Med. Toxicol., 9(1): 25-33.

Amptoulach S, Tsavaris N. 2011. Neurotoxicity caused by the treatment with platinum analogues. Chemother. Res. Pract., 2011: 1-5.

Arrieta Ó, Hernández-Pedro N, FernándezGonzález-Aragón MC, Saavedra-Pérez D, Campos-Parra AD, Ríos-Trejo MA, Cerón-Lizárraga T, Martínez-Barrera L, Pineda B, Ordóñez G, Ortiz-Plata A, Granados-Soto V, Sotelo J. 2011. Retinoic acid reduces chemotherapy induced neuropathy in an animal model and patients with lung cancer. Neurology, 77: 987-995.

Bancroft JD, Gamble M. 2008. Theory and Practice of Histology Techniques (6th edn). Churchill Livingstone, Elsevier: Philadelphia; 83 - 134.

Bottone MG, Santin G, Piccolin VM, Bo VD, Bernocchi G. 2012. Cisplatin neurotoxicity induces cell death in vitro and in vivo. In Cisplatin: Pharmacology, Clinical Uses and Adverse Effects, Kojima T, Morita Y (eds). NOVA Science Publishers, Inc.: New York; 123140.

Carozzi V, Chiorazzi A, Canta A, Oggioni N, Gilardini A, Rodriguez-Menendez V, Avezza F, Crippa L, Ceresa C, Nicolini G, Bossi M, Cavaletti G. 2009. Effect of the chronic combined administration of cisplatin and paclitaxel in a rat model of peripheral neurotoxicity. Eur. J. Cancer, 45: $656-665$.

Fahmy HA, Abd El-AzimeASh, Gharib OA. 2013. Possible ameliorative role of low dose of radiation against cisplatin induced oxidative stress and tissue damage in male rats. Eur. J. Bio. \& Med. Sci. Res., 1(4): 10-18.
Gregg RW, Molepo JM, Monpetit VJ, Mikael NZ, Redmond D, Gadia M, Stewart DJ. 1992. Cisplatin neurotoxicity: the relationship between dosage, time, and platinum concentration in neurologic tissues, and morphologic evidence of toxicity. Journal of Clinical Oncology, 10(5): 795-803.

Gulec M, Oral E, Dursun OB, Yucel A, Hacimuftuoglu A, Akcay F, Suleyman H. 2013. Mirtazapine protects against cisplatin-induced oxidative stress and DNA damage in the rat brain. Psychiatry and Clinical Neurosciences, 67: 50-58.

Hsiao G, Fong TH, Tzu NH, Lin KH, Chou DS, Sheu JR. 2004. A potent antioxidant, Lycopene, affords neuroprotection against microglia activation and focal cerebral ischemia in rats. In Vivo, 18: 351-356.

IARC (International Agency for Research on Cancer). 1981. Cisplatin, In Some Antineoplastic and Immunosuppressive Agents: IARC Monographs on the Evaluation of Immunosuppressive Agents. IARC: Lyon, France; 26: 151-164.

Ilbey YO, Ozbek E, Cekmen M, Simsek A, Otunctemur A, Somay A. 2009. Protective effect of curcumin in cisplatin induced oxidative injury in rat testis: Mitogen-activated protein kinase and nuclear factor-kappa B signaling pathways. Hum. Reprod, 24: 1717-1725.

Kim HJ, Lee JH, Kim SJ, Oh GS, Moon HD, Kwon KB, Park C, Park BH, Lee HK, Chung SY, Park R, So HS. 2010. Roles of NADPH oxidases in cisplatin-induced reactive oxygen species generation and ototoxicity. J. Neurosci, 30: 3933-3946.

Kreider JC, Blumberg MS. 1999. Geotaxis in 2-week-old Norway rats (Rattusnorvegicus): A reevaluation. Dev Psychobiol., 35(1):35-42.

Mckeage MJ, Hsu T, Screnci D, Haddad G, Baguley BC. 2001. Nucleolar damage correlates with neurotoxicity induced by different platinum drugs. Br. J. Cancer, 85: 1219-1225. 
McWhinney SR, Goldberg RM, Mcleod HL. 2009. Platinum neurotoxicity pharmacogenetics. Mol. Cancer Ther., 8: $10-16$.

Mohammad S, Shahrnaz P, Masoud N, Moazamehosadat R, Nasser Z, Khadije E, Fatemeh A. 2010. Walnut consumption protects rats against cisplatin-induced neurotoxicity. Neurotoxicology, 33(5): 1314-1321.

Nowis D, Legat M, Bil J, Kurzaj Z, Issat T, Stoklosa T, Mioduszewska B, Kaczmarek L, Jakóbisiak M, Golab J. 2007. Erythropoietin reduces cisplatin-induced neurotoxicity without impairment of cytotoxic effects against tumor cells. Int. J. Oncol., 31(6): 1547-1552.

Pandir D, Kara Ö. 2013. Cisplatin-induced kidney damage and the protective effect of bilberry (Vaccinium myrtillus L.): an experimental study. Turk. J. Med. Sci., 43: 951-956.

PHS (Public Health Service). 1996. Public Health Service Policy on Humane Care and Use of Laboratory Animals. US Department of Health and Human Services: Washington, DC, PL; 99-158.

Pisu MB, Roda E, Avella D, Bernocchi G. 2004. Developmental plasticity of rat cerebellar cortex after cisplatin injury: inhibitory synapses and differentiating Purkinje neurons. Neuroscience, 129: 655-664.

Pisu MB, Roda E, Guioli S, Avella D, Bottone MG, Bernocchi G. 2005. Proliferation and Migration of Granule Cells in the Developing Rat Cerebellum: Cisplatin
Effects. The Anatomical Record, 287A:1226-1235.

Prabhu VV, Kannan N, Guruvayoorappan C. 2013. 1,2-Diazole prevents cisplatininduced nephrotoxicity in experimental rats. Pharmacological Reports, 65: 980 990.

Stahl W, Sies H. 2003. Antioxidant activity of carotenoids. Mol. Aspects Med., 24(6): 345-351.

Turan MI, Turan IS, Mammadov R, Altinkaynak K, Kisaoglu A. 2013. The Effect of Thiamine and Thiamine Pyrophosphate on Oxidative Liver Damage Induced in Rats with Cisplatin. Bio. Med. Research International, Article ID 783809. 1-6.

Van Wijk N, Rijntjes E, van de Heijning BJ. 2008. Perinatal and chronic hypothyroidism impairs behavioural development in male and female rats. Exp Physiol., 93(11):1199-209.

Vivar C, vanPraag H. 2013. Functional circuits of new neurons in the dentate gyrus. Front Neural Circuits, 7(15): 1-13.

Wattanathorn J, Thukham-mee W, Muchimapura S, Tong-Un T, Wannanon P. 2012. Tomato, a Potential Yin Food, Protects against Stroke. Chinese Medicine, 3: 144-150. 